\title{
Oxalsäureverlust bel der Gefriertrocknung von oxalatreichen Pflanzen
}

Für die Konservierung von Lebensmitteln wurde in den letzten Jahren das Verfahren der Gefriertrocknung entwickelt. Sie wird heute schon industriell angewendet $\left.{ }^{1}\right), 2$ ). Bei der Gefriertrocknung erfolgt der Wasserentzug durch Sublimation. Es ist damit zu rechnen, daB bei diesem Vorgang auch Inhaltsstoffe aus den Nahrungsmitteln entfernt werden können. Dieses kann für die Wiederverwendung des Trockengutes sowohl von positiver als auch von negativer Bedeutung sein, je nachdem, ob es sich um Verluste von wertvollen Aromaund Wirkstoffen handelt oder von schädlichen und ungenießbaren Stoffen, wie $z$. B. Oxalsäure, Terpentin u.a.

Mit Hilfe einer Gefriertrocknungspilotanlage (EpsilonSonderausführung der Firma M. Christ, Osterode) wurden die während der Gefriertrocknung auftretenden Verluste ermittelt $\left.{ }^{3}\right)$. Es wurde festgestellt, daß während dieser Behandlung bei oxalatreichen Pflanzen der Oxalatgehalt signifikant abnimmt.

Um die Frage zu klären, welche Oxalatverbindungen (gelöste oder ungelöste Oxalate) bei der Gefriertrocknung verlorengehen, wurden die reine Oxalsäure und ihre Natrium- und Calciumsalze untersucht (Fig. 1). Hierbei stellte sich eine minimale Gewichtsabnahme von Natriumund Calciumoxalat heraus, das wohl auf Spuren von Wasser zurückzuführen ist. Dagegen liegen die Oxalsäureverluste erheblich höher und sind abhängig von der Trocknungsdauer. Eine vollkommene Sublimation tritt bei genügend langer Gefriertrocknung ein.

Auf Grund der unterschiedlichen Sublimationseigenschaften ist es möglich, den Oxalsäureanteil in einem Gemisch von gelösten Oxalaten (Oxalsäure und lösliche Salze) zu bestimmen, wenn durch Gefriertrocknung die leicht sublimierbare Säure aus dem Gemisch entfernt wird.

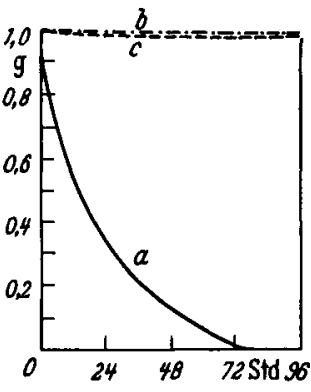

Fig. 1. Verănderung des Gewichts verschiedener Oxalate während der Gefriertrocknung. (TablarTemperatur $+30^{\circ} \mathrm{C}$, Vorkühlung $-30^{\circ} \mathrm{C}$, Vakuum 0,2 Torr.) a Oxalsäure; $b$ Na-Oxalat; $c$ Ca-Oxalat 
Wir danken der Deutschen Forschungsgemeinschaft für die finanzielle Unterstützung dieser Arbeit.

Max-Planck-Institut füx Kulturpflanzenzüchtung, HamburgVolksdorf

G. Kallistratos u. R. v. Sengbusch

Eingegangen am 29. Februar 1964

1) Freeze-Drying of Foods. Chicago, Illinois 1961. - 2) $5 . \mathrm{Ge}-$ friertrocknungstagg. Köln: Leybold 1962. ${ }^{3}$ ) KALListratos, G., u. R. v. SENGBusch: In Vorbereitung. 